ARTICLE

DOI: $10.1038 / \mathrm{s} 41467-018-02899-5$

OPEN

\title{
A peak in the critical current for quantum critical superconductors
}

Soon-Gil Jung (1D ${ }^{1}$, Soonbeom Seo ${ }^{1,2}$, Sangyun Lee ${ }^{1}$, Eric D. Bauer (1) ${ }^{2}$, Han-Oh Lee ${ }^{3}$ \& Tuson Park ${ }^{1}$

Generally, studies of the critical current $I_{\mathrm{C}}$ are necessary if superconductors are to be of practical use, because $I_{c}$ sets the current limit below which there is a zero-resistance state. Here, we report a peak in the pressure dependence of the zero-field $I_{c}, I_{c}(0)$, at a hidden quantum critical point (QCP), where a continuous antiferromagnetic transition temperature is suppressed by pressure toward $O \mathrm{~K}$ in CeRhIn $n_{5}$ and $4.4 \% \mathrm{Sn}$-doped CeRhln $\mathrm{n}_{5}$. The $I_{\mathrm{c}}(0) \mathrm{s}$ of these Ce-based compounds under pressure exhibit a universal temperature dependence, underlining that the peak in zero-field $I_{c}(P)$ is determined predominantly by critical fluctuations associated with the hidden QCP. The dc conductivity $\sigma_{\mathrm{dc}}$ is a minimum at the QCP, showing anti-correlation with $I_{c}(0)$. These discoveries demonstrate that a quantum critical point hidden inside the superconducting phase in strongly correlated materials can be exposed by the zero-field $I_{\mathrm{C}}$, therefore providing a direct link between a QCP and unconventional superconductivity.

\footnotetext{
${ }^{1}$ Center for Quantum Materials and Superconductivity (CQMS), Department of Physics, Sungkyunkwan University, Suwon 16419, South Korea. ${ }^{2}$ Los Alamos National Laboratory, Los Alamos, NM 87545, USA. ${ }^{3}$ Center for Correlated Matter and Department of Physics, Zhejiang University, 310058 Hangzhou, China. Correspondence and requests for materials should be addressed to T.P.tp8701@skku.edu)
} 
nconventional superconductivity (SC) often is observed in close proximity to a magnetically ordered phase, where the SC transition temperature $T_{\mathrm{c}}$ forms a dome against a non-thermal control parameter, such as the external pressure, chemical substitution, or magnetic field ${ }^{1-6}$. At an optimal value of the tuning parameter, where $T_{c}$ is the highest, normal state properties do not follow predictions for Landau-Fermi liquids: the electrical resistivity $(\rho)$ does not exhibit a $T^{2}$ dependence, and the electronic specific heat coefficient $(\gamma=C / T)$ does not saturate, but rather diverges with decreasing temperature ${ }^{1,2,7}$. These nonFermi liquid (NFL) behaviors arise from incoherent critical fluctuations associated with a quantum critical point (QCP) hidden inside the SC dome of heavy fermion compounds and some Fe-based superconductors, such as $\mathrm{BaFe}_{2}\left(\mathrm{As}_{1}\right.$ $\left.{ }_{-x} \mathrm{P}_{x}\right)_{2}{ }^{1,2}, 4,6,8$. Because the zero-temperature quantum phase transition is typically not accessible without destroying SC, the role of critical magnetic fluctuations on properties of unconventional superconductors has yet to be explored in depth.

The critical current $\left(I_{c}\right)$, which limits the current capacity of a zero-resistance state, is characteristically taken to depend on the strength of vortex pinning, which, in turn, is determined by the geometry and distribution of microstructural defects ${ }^{9-11}$. Because application of pressure should not lead to the creation of different or additional defects or to a substantial change in sample dimensions, $I_{c}$ in relation to $T_{c}$ should be at most weakly pressure-dependent. A substantial variation in $I_{c}(P)$ or $I_{\mathrm{c}} / T_{\mathrm{c}}(P)$, then, logically, should be attributed to intrinsic changes in the superconducting state itself. For example, the zero-field critical current density $J_{c}$ (equal to $I_{c} / A$, where $A$ is the sample cross sectional area perpendicular to current) of the hole-doped high$T_{c}$ cuprate superconductor $\mathrm{Y}_{0.8} \mathrm{Ca}_{0.2} \mathrm{Ba}_{2} \mathrm{Cu}_{3} \mathrm{O}_{y}$ has a sharp peak that is centered on a critical hole-doping where the pseudogap boundary line projects to zero temperature, and that is attributed in model calculations to changes in the superfluid density ${ }^{12,13}$. These results indicate that $I_{c}$ measurements may provide an opportunity to explore the relationship between unconventional SC and any QCP that is hidden beneath the SC dome.

Here we report a peak in the zero-field critical current, $I_{c}(0)$, at a critical pressure $P_{c}$ in pure $\mathrm{CeRhIn}_{5}$ (Rh115) and $4.4 \% \mathrm{Sn}$ doped $\mathrm{CeRhIn}_{5}$ (SnRh115), where their respective antiferromagnetic boundary $T_{\mathrm{N}}(P)$ extrapolates to $T=0 \mathrm{~K}$ inside a dome of pressure-induced SC. The temperature dependence of $I_{c}(0)$ s for pure Rh115 and SnRh115 under pressure is similar to that of superconducting $\mathrm{CeCoIn}_{5}$, which is close to quantum criticality at ambient pressure. Normalized values of $I_{\mathrm{c}}(T, P)$ follow a common universal curve for each material, suggesting an intrinsic, fundamental connection to quantum criticality. Supporting this conclusion, the magnetic field dependence of the flux-pinning force $\left(F_{\mathrm{p}}=I_{\mathrm{c}} \times \mu_{0} H\right)$, normalized to its maximum value, also forms a pressure-invariant universal curve for each compound. As will be discussed, these discoveries demonstrate that the pressure evolution of zero-field $I_{\mathrm{c}}$ is determined mainly by quantum critical fluctuations, and that the peak in $I_{c}$ is a direct link to the hidden QCP.

\section{Results}

Temperature-pressure phase diagrams. Figure $1 \mathrm{a}$ and $\mathrm{b}$ presents a contour plot of the zero-field $I_{\mathrm{c}}(P, T)$ in the SC phase and the in-plane resistivity $\rho_{\mathrm{ab}}(P, T)$ in the normal state for pure CeRhIn (Rh115) and $4.4 \%$ Sn-doped $\mathrm{CeRhSn}_{0.22} \mathrm{In}_{4.78}$ (SnRh115) single crystals. The dependence on pressure of the in-plane resistivity and current-voltage curves upon which Fig. 1 is based is displayed in Supplementary Figs. 1 and 2, respectively. The quantum critical region veiled by the superconducting phase is fully exposed by the pressure dependence of zero-field $I_{c}(T)$. A sharp
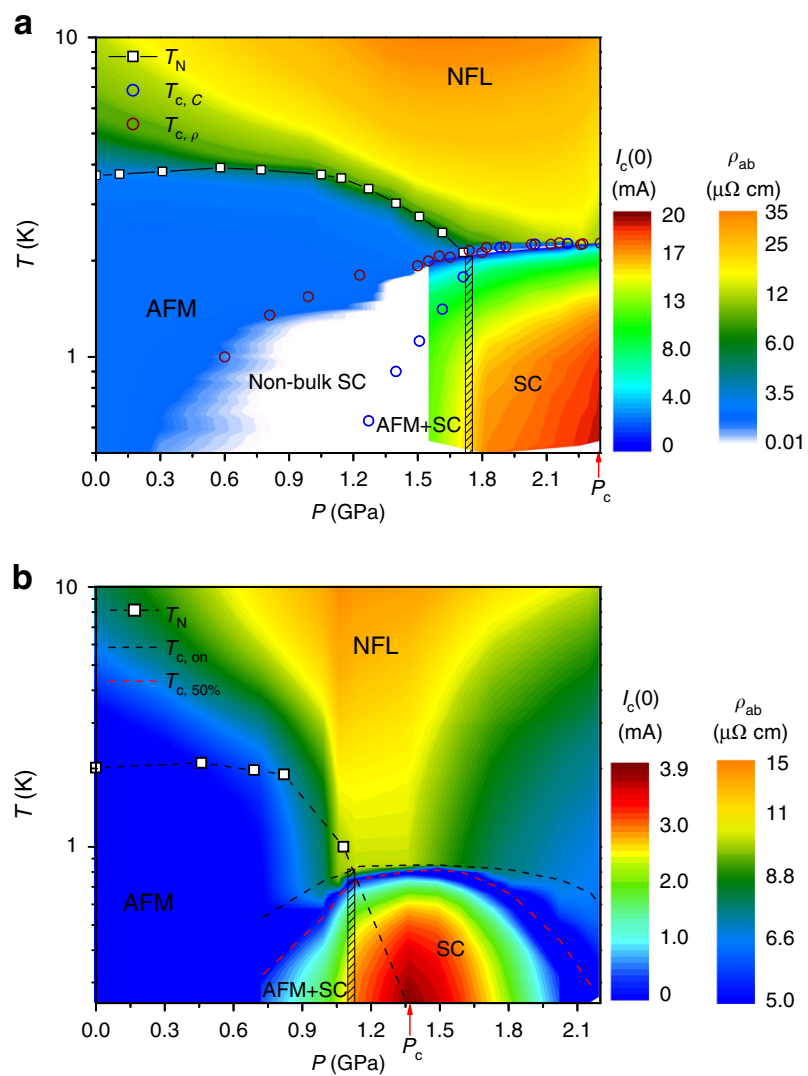

Fig. 1 Temperature-pressure phase diagrams of CeRhln $n_{5}$ and CeRhSn $0.22 \ln _{4.78}$ single crystals. In the superconducting state below $T_{c}(P)$ false colors denote the magnitude of the zero-field critical current $I_{c}(P, T)$. At temperatures above $T_{\mathrm{c}}(P)$, false colors reflect the magnitude of the inplane resistivity $\rho_{\mathrm{ab}}(P, T)$. a CeRhln ${ }_{5}(\mathrm{Rh} 115)$ and $\mathbf{b} \mathrm{CeRhSn} 0.22 \mathrm{ln} 4.78$ (SnRh115). For both materials, $\rho_{\mathrm{ab}}(P, T)$ is enhanced around the quantum critical point $P_{\mathrm{C}}$ due to pronounced incoherent inelastic scattering. Similarly, the zero-field $I_{c}(P, T)$ is largest at $P_{c}$, where the QCP is expected, as indicated by the arrow. In both $\mathbf{a}$ and $\mathbf{b}$ the vertical hashed rectangle is at $P_{c}{ }^{\star}$, the pressure that separates a phase of coexisting superconductivity and magnetism from a purely $\mathrm{SC}$ phase for $P>P_{\mathrm{C}}{ }^{\star}$. Open squares in both $\mathbf{a}$ and b represent the antiferromagnetic transition temperature $\left(T_{N}\right)$. SC transition temperature $\left(T_{\mathrm{C}}\right)$ of Rh115 is evaluated from specific heat $\left(T_{\mathrm{C}, C}\right)$ and resistivity $\left(T_{c, \rho}\right)$ measurements, and $T_{\mathrm{c}}$ of SnRh115 is determined as $T_{\mathrm{c}}$ onset $\left(T_{c, 0 n}\right)$ and $50 \%\left(T_{c, 50 \%}\right)$ of the normal state resistivity value at $T_{c, o n}$. AFM, SC, and NFL stand for antiferromagnetic, superconducting, and nonFermi liquid regions, respectively

peak in the value of $I_{c}(T)$ is clearly observed for pressures around the QCP at $P_{\mathrm{c}}$, where a large enhancement in the resistivity is accompanied by strong quantum fluctuations ${ }^{3,4,14}$. In addition, $I_{c}(P)$ abruptly increases at pressures around $P_{c}^{*}$, the critical pressure where coexisting phases of magnetism and SC evolve into a single SC state. In undoped Rh115, large differences between $T_{c} s$ measured by heat capacity $(C)$ and resistivity $(\rho)$ at pressures below $P_{c}^{*}$ are ascribed to textured SC originating from an incommensurate long-range magnetic order ${ }^{15-18}$.

Temperature dependences of the zero-field critical current. The antiferromagnetic transition temperature $\left(T_{\mathrm{N}} \sim 3.8 \mathrm{~K}\right)$ in pure Rh115 is suppressed by Sn doping, which induces a shift of its extrapolated $T=0 \mathrm{~K}$ antiferromagnetic transition, and pressureinduced superconductivity emanates from the tuned QCP ${ }^{4,5}$ (see Supplementary Fig. 3). Figure $2 \mathrm{a}$ and $\mathrm{b}$ shows the temperature 

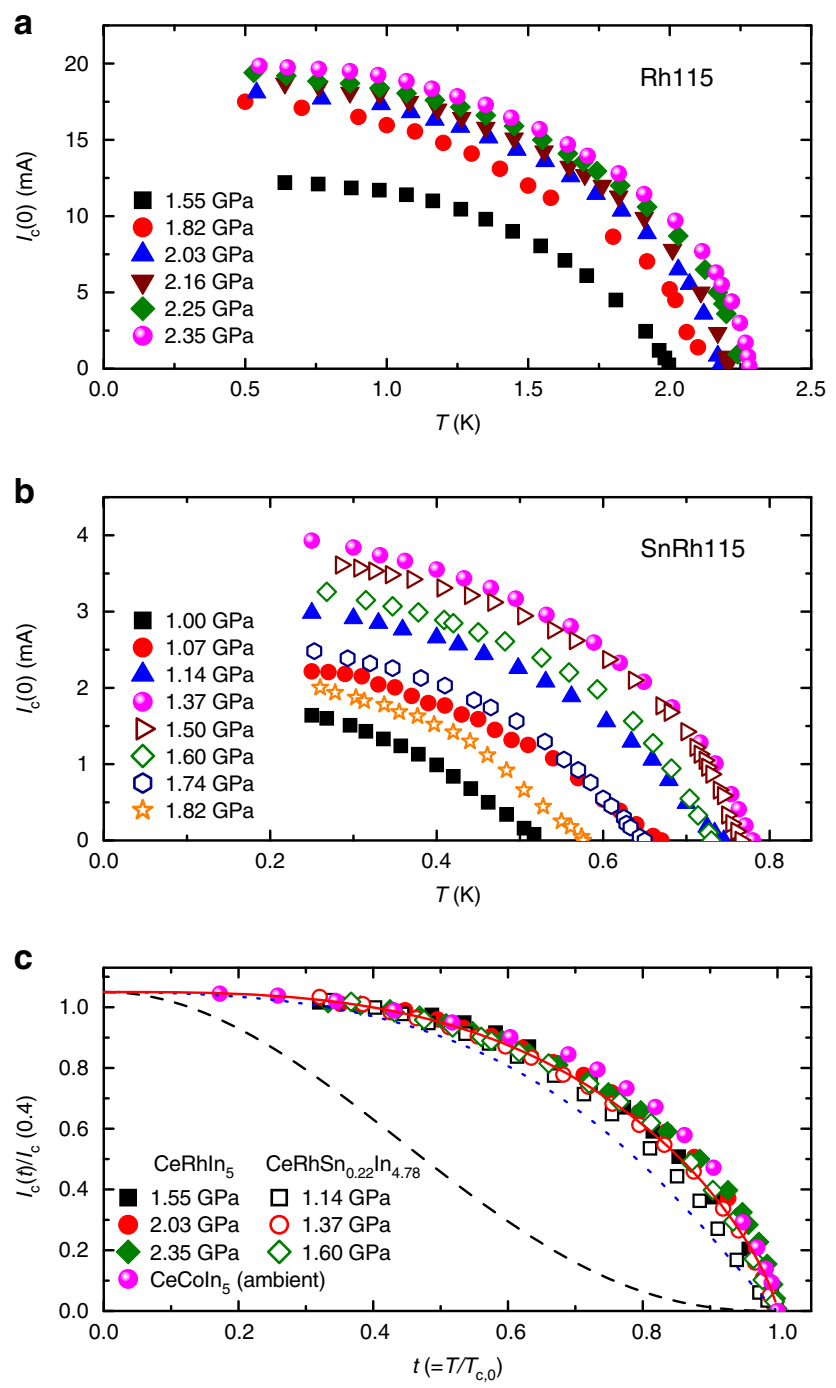

Fig. 2 Temperature dependences of the zero-field critical current for $\mathrm{Ce}$ based heavy fermion materials under pressure. a Temperature dependence of the zero-field critical current, $I_{c}(0)$, for $\mathrm{CeRh} \mathrm{n}_{5}$ at various pressures. $\mathbf{b}$ Zero-field $I_{\mathrm{c}}$ for $\mathrm{CeRhSn}_{0.22} \mathrm{In}_{4.78}$ at various pressures. c Reduced temperature $\left(t=T / T_{\mathrm{c}, 0}\right)$ dependence of $I_{\mathrm{c}} I_{\mathrm{c}}(t)$, normalized by its value at $t$ $=0.4$ for Rh115 and SnRh115 at representative pressures and for CeColn ${ }_{5}$ at ambient pressure. The normalized values of $I_{c}(P, t)$ for all crystals can be described by a single curve, $I_{c}(t) \propto\left(1-t^{2}\right)^{5 / 6}\left(1+t^{2}\right)^{2 / 3}$, indicating universal behavior of $I_{c}(t)$ with respect to pressure in the CeMln ${ }_{5}(M=C o, R h)$ materials. Dotted and dashed curves are for $\delta T_{c}$-pinning and $\delta /$-pinning, respectively, as discussed in the text

dependence of the zero-field critical current, $I_{\mathrm{c}}(0)$, for Rh115 and $\mathrm{SnRh} 115$ at several pressures, respectively. Here, $I_{\mathrm{c}}$ is determined by using the voltage criterion of $0.1 \mu \mathrm{V}$ (see Supplementary Fig. 2). Analysis of the flux-pinning force, $F_{\mathrm{p}}=I_{\mathrm{c}} \times \mu_{0} H$, shows that the normalized flux-pinning force follows a power-law dependence on magnetic field, $f_{\mathrm{p}}(h) \propto h^{p}(1-h)^{q}$, and is peaked around $h_{\text {peak }} \approx 0.6$, which is characteristic of type-II superconductors with weak pinning (see Supplementary Fig. 4). Here, the normalized pinning force is $f_{\mathrm{p}}=F_{\mathrm{p}} / F_{\mathrm{p}, \max }$ and the reduced field is $h=H / H_{\text {irr }}$, where $F_{\mathrm{p} \text {,max }}$ is the maximum flux-pinning force and $H_{\text {irr }}$ is the irreversible field. The dependence on temperature of the critical current has been widely explained by $I_{\mathrm{c}}(t) /$ $I_{\mathrm{c}}(0)=\left(1-t^{2}\right)^{\alpha}\left(1+t^{2}\right)^{\beta}$ for type-II superconductors, such as high$T_{\mathrm{c}}$ cuprates, Fe-based superconductors, and $\mathrm{MgB}_{2}{ }^{19-22}$, where $t=$ $T / T_{\mathrm{c}}$ is the reduced temperature. When $T_{\mathrm{c}}$ variations surrounding defects are important ( $\delta T_{\mathrm{c}}$-pinning), $\alpha=7 / 6$ and $\beta=5 / 6$, but $\alpha=$ $5 / 2$ and $\beta=-1 / 2$ for $\delta l$-pinning that arises from spatial variations in the charge-carrier mean free path $(l)$ near a lattice defect $^{10,19,23}$. These functional forms are shown by the dotted and dashed lines for $\delta T_{\mathrm{c}}$-pinning to $\delta l$-pinning in Fig. $2 \mathrm{c}$, respectively. A crossover of the mechanism from $\delta T_{\mathrm{c}}$-pinning to $\delta l$-pinning has been often reported by introducing additional defects via chemical substitution or heavy ion irradiation, indicating that $\delta T_{\mathrm{c}}$-pinning is preferred in clean crystals ${ }^{19-21}$.

The values of $I_{c}(t)$ for $\operatorname{SnRh} 115$ at pressures around $P_{c}$ are fitted together with those for Rh115 and CeCoIn ${ }_{5}$ in Fig. 2c. The temperature dependence of $I_{c}(0)$ for $\mathrm{CeCoIn}_{5}$ at ambient pressure is measured to compare it with that of CeRhIn , because Rh115 is believed to have a SC pairing mechanism similar to that in $\mathrm{CeCoIn}_{5}$. The values of $I_{c}(0)$ for all samples can be expressed well by one curve with the relation $I_{c}(t) \propto\left(1-t^{2}\right)^{5 / 6}\left(1+t^{2}\right)^{2 / 3}$, which is distinct from that for $I_{\mathrm{c}}(t)$ controlled by either $\delta T_{\mathrm{c}}$-pinning or $\delta l$ pinning. This universal curve underscores that the origin of the zero-field $I_{\mathrm{c}}$ is the same for each compound, and that it does not change under pressure for these Ce-based quantum critical materials. The fact that external pressure does not create new defects inside the crystals suggests that the pressure evolution of $I_{c}$ should be related to the pressure dependence of the SC coupling strength.

\section{Discussion}

Figure 3a presents the pressure dependences of $I_{\mathrm{c}}(0)$ and $T_{\mathrm{c}, 0}$ for SnRh115, which are similar to each other. However, their relative fractional variations in $I_{\mathrm{c}}$ and $T_{\mathrm{c}}, \gamma_{I} \equiv I_{\mathrm{c}, 0}(P) / I_{\mathrm{c}, 0}\left(P_{\mathrm{c}}\right) \times 100$ and $\gamma_{T} \equiv T_{\mathrm{c}, 0}(P) / T_{\mathrm{c}, 0}\left(P_{\mathrm{c}}\right) \times 100$, where $I_{\mathrm{c}, 0}\left(P_{\mathrm{c}}\right)$ is $I_{\mathrm{c}}$ extrapolated to zero temperature at $P_{\mathrm{c}}$ and $T_{\mathrm{c}, 0}\left(P_{\mathrm{c}}\right)$ is the SC transition temperature at $P_{\mathrm{c}}$, are much different, as shown in Fig. $3 \mathrm{~b}$ : at $1.0 \mathrm{GPa}$, the critical current is $45 \%$ of the maximum value, and $T_{\mathrm{c}, 0}$ is $67 \%$ of its maximal value. The stronger pressure dependence of $I_{\mathrm{c}}$ relative to that of $T_{\mathrm{c}, 0}$ is clearly visible in ratio $I_{\mathrm{c}, 0} / T_{\mathrm{c}, 0}$ for $S n R h 115$, as presented in Fig. $3 c$, d. An abrupt enhancement in $I_{\mathrm{c}, 0} / T_{\mathrm{c}, 0}$ is observed at $P_{\mathrm{c}}^{*}$, and the peak in the pressure dependence of $I_{\mathrm{c}, 0} /$ $T_{\mathrm{c}, 0}$ is achieved at $P_{\mathrm{c}}$. The dc conductivity $\left(=\sigma_{\mathrm{dc}}\right)$ at $T_{\mathrm{c}}$ onset is shown as a function of pressure in the right ordinate of Fig. $3 c$, where a minimum value appears near $P_{c}$ (see Supplementary Fig. 5). The anti-correlation between $I_{c, 0} / T_{\mathrm{c}, 0}$ and $\sigma_{\mathrm{dc}}$ in these Cebased quantum critical compounds may be related with the presence of the hidden QCP at $P_{\mathrm{c}}$, because the associated critical quantum fluctuations not only act as the SC pairing glue, but also strongly enhance incoherent electron scattering, thus leading to a minimum in $\sigma_{\mathrm{dc}}$ at $P_{\mathrm{c}}^{24,25}$. Homes' scaling relation ${ }^{26-28}$ states that the superfluid density $n_{\mathrm{s}}$ is proportional to $\sigma_{\mathrm{dc}} T_{\mathrm{c}}$ in many correlated superconductors and, consequently, that the ratio $n_{s} / T_{c}$ should be proportional to $\sigma_{\mathrm{dc}}$. The fact that $\sigma_{\mathrm{dc}}$ is the minimum at $P_{\mathrm{c}}$, where $I_{\mathrm{c}, 0} / T_{\mathrm{c}, 0}$ is the maximum in these Ce-based compounds, suggests a violation of Homes' scaling if the strength of the condensate $n_{\mathrm{s}}$ is proportional to the critical current $I_{\mathrm{c}, 0}$. Pressuredependent optical conductivity and/or penetration depth experiments that directly measure $n_{\mathrm{s}}$ will be important to provide a stringent test for the validity of Homes' law in quantum critical superconductors.

Our study demonstrates that the critical current, a fundamental superconducting parameter, is a powerful tool for investigating the presence of a hidden QCP inside the superconducting dome without destroying the superconducting phase. The dependence on temperature of the zero-field $I_{\mathrm{c}}$ for both pure Rh115 and Sndoped Rh115 exhibits the same functional form under pressure, underscoring that the peak at $P_{\mathrm{c}}$ in the pressure dependence of $I_{\mathrm{c}}$ arises from an enhanced fluctuations around the hidden QCP. Even though these results are specific to the Ce115 heavy-fermion 

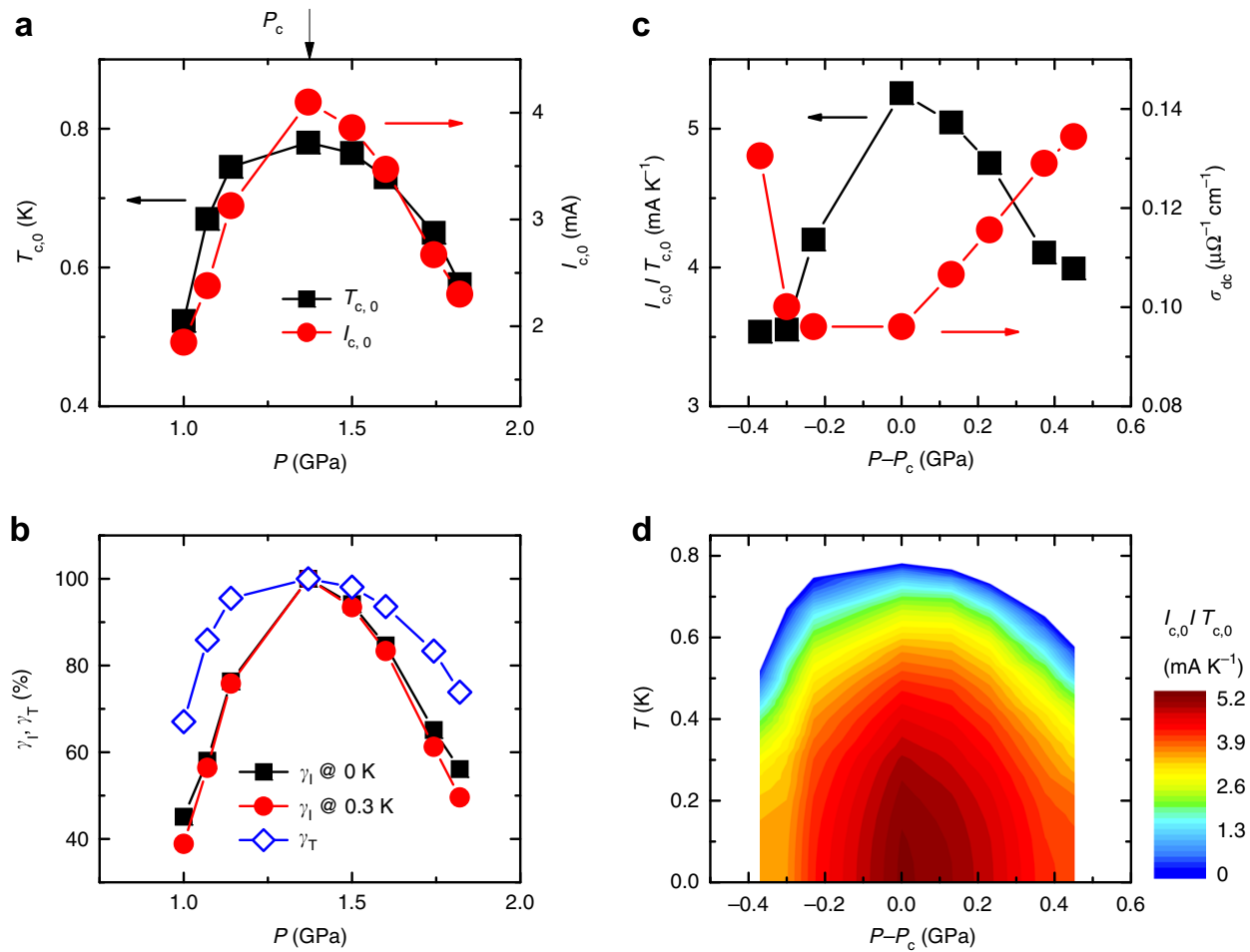

Fig. 3 Pressure evolution of the zero-field critical current in Sn-doped CeRh $n_{5}$. a Pressure dependences of $T_{c, 0}$ and $I_{c, 0}$ for $\mathrm{SnRh} 115$, where $I_{c, 0}$ is the value of $I_{\mathrm{c}}$ obtained from an extrapolation of data in Fig. $2 \mathrm{~b}$ zero Kelvin. $\mathbf{b}$ Fractional variations in $I_{\mathrm{c}, \mathrm{O}}$ and $T_{\mathrm{c}, \mathrm{O}}$ for SnRh115 under pressure. The fractions are defined as $\gamma_{l}($ at $0 \mathrm{~K}) \equiv I_{\mathrm{c}, 0}(P) / I_{\mathrm{c}, 0}\left(P_{\mathrm{c}}\right) \times 100$ and $\gamma_{T} \equiv T_{\mathrm{c}, 0}(P) / T_{\mathrm{c}, 0}\left(P_{\mathrm{c}}\right) \times 100$, where $I_{\mathrm{c}, 0}\left(P_{\mathrm{c}}\right)$ is $I_{\mathrm{c}}$ extrapolated to zero temperature at $P_{\mathrm{c}}$ and $T_{\mathrm{c}, 0}\left(P_{\mathrm{c}}\right)$ is the superconducting transition temperature at $P_{\mathrm{c}}$. Values of $\gamma_{l}$ are plotted as a function of pressure for measured or estimated $I_{\mathrm{c}}(T, P)$ at $0 \mathrm{~K}(\mathrm{squares})$ and 0.3 $\mathrm{K}$ (circles). c The ratio between the critical current and SC transition temperature, $I_{\mathrm{c}, \mathrm{O}} / T_{\mathrm{c}, 0}$, plotted together with the dc conductivity at $T_{\mathrm{c}}$ onset, $\sigma_{\mathrm{dc}}$, as a function of the pressure difference $P-P_{\mathrm{c}}$, where $P_{\mathrm{c}}=1.35 \mathrm{GPa}$ is the QCP. $\mathbf{d}$ A contour plot of $I_{\mathrm{c}, \mathrm{O}} / T_{\mathrm{c}, \mathrm{O}}$ displayed in the temperature $(T)$ and pressure $\left(P-P_{\mathrm{c}}\right)$ plane. The ratio $I_{\mathrm{c}, \mathrm{O}} / T_{\mathrm{c}, \mathrm{O}}$ forms a dome centered around the quantum critical point $P_{\mathrm{c}}$ and its values decrease with distance from $P_{\mathrm{c}}$

materials, the prediction of similar results for the hole-doping dependence of the critical current density $J_{c}(x)$ in high- $T_{c}$ cuprates $^{29}$ suggests a universal behavior of $J_{c}$ among unconventional superconductors. These discoveries should stimulate more theoretical and experimental effort to understand the intimate link between quantum criticality and the origin of unconventional superconductivity in various families of correlated electronic systems.

\section{Methods}

Measurement outline. CeRhIn ${ }_{5}$, Sn-doped $\mathrm{CeRhIn}_{5}$, and $\mathrm{CeCoIn}_{5}$ single crystals were synthesized by the indium (In) self-flux method ${ }^{30-32}$. Pressure was generated in a hybrid clamp-type pressure cell with Daphne 7373 as the pressure-transmitting medium, and the pressure was determined by monitoring the shift in the value of $T_{\mathrm{c}}$ for lead $(\mathrm{Pb})$. Measurements of current-voltage $(I-V)$ characteristics under pressure were performed in a Heliox VL system (Oxford Instruments) with a vector magnet ( $y=5 \mathrm{~T}$ and $z=9 \mathrm{~T}$, American Magnetics Inc.) and in a Physical Property Measurement System (PPMS 9 T, Quantum Design), where the current was provided by a Keithley 6221 unit and the voltage was measured with a Keithley 2182A nanovoltmeter.

Measurement details. Measurements of $I-V$ characteristics were performed in a pulsed mode to minimize Joule heating developed at Ohmic contacts to the samples and copper $(\mathrm{Cu})$ wires between the pressure cell and the connector. The duration of the pulsed current was $10-11 \mathrm{~ms}$, and the repetition rate was one pulse every $2 \mathrm{~s}$, which was sufficient to eliminate Joule heat in the samples ${ }^{33,34}$. A standard four-probe method was used to determine $I-V$, and good Ohmic contact to samples was achieved by using silver epoxy. The critical current was based on a $10^{-7} \mathrm{~V}$ criterion ${ }^{35}$, which was averaged over three measurements. The dimensions of the measured crystals were $920 \times 330 \times 20,650 \times 200 \times 22$, and $1100 \times 200 \times 47 \mu^{3}$ for $\mathrm{CeRhIn}_{5}(\mathrm{Rh} 115), \mathrm{CeRhSn}_{0.22} \mathrm{In}_{4.78}(\mathrm{SnRh115})$, and CeCoIn ${ }_{5}$, respectively. The magnetic-field dependence of the critical current was measured at several pressures and the flux-pinning force $\left(F_{\mathrm{p}}\right)$ was estimated from the relation $F_{\mathrm{p}}=I_{\mathrm{c}} \times \mu_{0} H^{36-38}$
Data availability. The data sets generated and/or analyzed in this study are available from the corresponding author on reasonable request.

Received: 31 May 2017 Accepted: 8 January 2018

Published online: 30 January 2018

\section{References}

1. Park, T. et al. Hidden magnetism and quantum criticality in the heavy fermion superconductor CeRhIn 5 . Nature 440, 65-68 (2006).

2. Knebel, G., Aoki, D., Braithwaite, D., Salce, B. \& Flouquet, J. Coexistence of antiferromagnetism and superconductivity in $\mathrm{CeRhIn}_{5}$ under high pressure and magnetic field. Phys. Rev. B 74, 020501(R) (2006).

3. Park, T. et al. Isotropic quantum scattering and unconventional superconductivity. Nature 456, 366-368 (2008).

4. Seo, S. et al. Controlling superconductivity by tunable quantum critical points. Nat. Commun. 6, 6433 (2015).

5. Ferreira, L. M. et al. Tuning the pressure-induced superconducting phase in doped CeRhIn ${ }_{5}$. Phys. Rev. Lett. 101, 017005 (2008).

6. Analytis, J. G. et al. Transport near a quantum critical point in $\mathrm{BaFe}_{2}\left(\mathrm{As}_{1}\right.$ $\left.{ }_{x} \mathrm{P}_{x}\right)_{2}$. Nat. Phys. 10, 194-197 (2014).

7. Gegenwart, P., Si, Q. \& Steglich, F. Quantum criticality in heavy-fermion metals. Nat. Phys. 145, 186-197 (2008).

8. Hashimoto, K. et al. A sharp peak of the zero-temperature penetration depth at optimal composition in $\mathrm{BaFe}_{2}\left(\mathrm{As}_{1-x} \mathrm{P}_{x}\right)_{2}$. Science 336, 1554-1557 (2012).

9. Fang, L. et al. Huge critical current density and tailored superconducting anisotropy in $\mathrm{SmFeAsO}_{0.8} \mathrm{~F}_{0.15}$ by low-density columnar-defect incorporation. Nat. Commun. 4, 2655 (2013)

10. Kunchur, M. N., Lee, S.-I. \& Kang, W. N. Pair-breaking critical current density of magnesium diboride. Phys. Rev. B 68, 064516 (2003).

11. Dew-Hughes, D. The critical current of superconductors: an historical review. Low Temp. Phys. 27, 713-722 (2001).

12. Talantsev, E. F. \& Tallon, J. L. Universal self-field critical current for thin-film superconductors. Nat. Commun. 6, 7820 (2015). 
13. Talantsev, E. F., Crump, W. P. \& Tallon, J. L. Thermodynamic parameters of single- or multi-band superconductors derived from self-field critical currents. Ann. Phys. 529, 1700197 (2017).

14. Knebel, G., Aoki, D., Brison, J.-P. \& Flouquet, J. The quantum critical point in CeRhIn $_{5}$ : a resistivity study. J. Phys. Soc. Jpn. 77, 114704 (2008).

15. Park, T. et al. Textured superconducting phase in the heavy fermion CeRhIn 5 . Phys. Rev. Lett. 108, 077003 (2012)

16. Park, T. \& Thompson, J. D. Magnetism and superconductivity in strongly correlated CeRhIn ${ }_{5}$. New J. Phys. 11, 055062 (2009).

17. Llobert, A. et al. Magnetic structure of $\mathrm{CeRhIn}_{5}$ as a function of pressure and temperature. Phys. Rev. B 69, 024403 (2004).

18. Yashima, M. et al. Strong coupling between antiferromagnetic and superconducting order parameters of CeRhIn ${ }_{5}$ studied by ${ }^{115} \mathrm{In}$ nuclear quadrupole resonance spectroscopy. Phys. Rev. B 79, 214528 (2009).

19. Griessen, R. et al. Evidence for mean free path fluctuation induced pinning in $\mathrm{YBa}_{2} \mathrm{Cu}_{3} \mathrm{O}_{7}$ and $\mathrm{YBa}_{2} \mathrm{Cu}_{4} \mathrm{O}_{8}$ films. Phys. Rev. Lett. 72, 1910-1913 (1994).

20. Wen, H. H., Zhao, Z. X., Xiao, Y. G., Yin, B. \& Li, J. W. Evidence for flux pinning induced by spatial fluctuation of transition temperatures in single domain $\left(\mathrm{Y}_{1-x} \mathrm{Pr}_{x}\right) \mathrm{Ba}_{2} \mathrm{Cu}_{3} \mathrm{O}_{7-\delta}$ samples. Physica C 251, 371-378 (1995).

21. Xiang, F. X. et al. Evidence for transformation from $\delta T_{\mathrm{c}}$ to $\delta l$ pinning in $\mathrm{MgB}_{2}$ by graphene oxide doping with improved low and high field $J_{c}$ and pinning potential. Appl. Phys. Lett. 102, 152604 (2013).

22. Ghorbani, S. R., Wang, X. L., Shahbazi, M., Dou, S. X. \& Lin, C. T. Fluctuation of mean free path and transition temperature induced vortex pinning in (Ba, K)Fe $\mathrm{Fe}_{2}$ superconductors. Appl. Phys. Lett. 100, 212601 (2012).

23. Blatter, G., Feigel'man, M. V., Geshkenbein, V. B., Larkin, A. I. \& Vinokur, V. M. Vortices in high-temperature superconductors. Rev. Mod. Phys. 66, 1125 (1994).

24. Howald, L., Knebel, G., Aoki, D., Lapertot, G. \& Brison, J.-P. The upper critical field of CeCoIn ${ }_{5}$. New J. Phys. 13, 113039 (2011).

25. Miyake, K. \& Narikiyo, O. Enhanced impurity scattering due to quantum critical fluctuations: perturbational approach. J. Phys. Soc. Jpn. 71, 867-871 (2002).

26. Homes, C. C. et al. A universal scaling relation in high-temperature superconductors. Nature 430, 539-541 (2004).

27. Dordevic, S. V., Basov, D. N. \& Homes, C. C. Do organic and other exotic superconductors fail universal scaling relations? Sci. Rep. 3, 1713 (2013).

28. Uemura, Y. J. et al. Universal correlations between $T_{\mathrm{c}}$ and $n_{\mathrm{s}} / m^{*}$ (carrier density over effective mass) in high- $T_{\mathrm{c}}$ cuprate superconductors. Phys. Rev. Lett. 62, 2317-2320 (1989).

29. Tallon, J. L. et al. Critical doping in overdoped high- $T_{\mathrm{c}}$ superconductors: a quantum critical point? Phys. Stat. Sol. B 251, 531-540 (1999).

30. Hegger, H. et al. Pressure-induced superconductivity in quasi-2D CeRhIn ${ }_{5}$. Phys. Rev. Lett. 84, 4986-4989 (2000).

31. Bauer, E. D. et al. Antiferromagnetic quantum critical point in $\mathrm{CeRhIn}{ }_{5-x} \mathrm{Sn}_{x}$. Physica B 378-380, 142-143 (2006).

32. Petrovic, C. et al. Heavy-fermion superconductivity in $\mathrm{CeCoIn}_{5}$ at $2.3 \mathrm{~K}$. J. Phys.: Condens. Matter 13, L337-L342 (2001).

33. Kunchur, M. N. Current-induced pair breaking in magnesium diboride. J. Phys.: Condens. Matter 16, R1183-R1204 (2004).

34. Liang, M., Kunchur, M. N., Fruchter, L. \& Li, Z. Z. Depairing current density of infinite-layer $\mathrm{Sr}_{1-x} \mathrm{La}_{x} \mathrm{CuO}_{2}$ superconducting films. Physica C 492, 178-180 (2013).

35. Dobrovolskiy, O. V., Begun, E., Huth, M. \& Shklovskij, V. A. Electrical transport and pinning properties of $\mathrm{Nb}$ thin films patterned with focused ion beam-milled washboard nanostructures. New J. Phys. 14, 113027 (2012).
36. Fietz, W. A. \& Webb, W. W. Hysteresis in superconducting alloystemperature and field dependence of dislocation pinning in niobium alloys. Phys. Rev. 178, 657-667 (1969).

37. Dew-Hughes, D. Flux pinning mechanisms in type-II superconductors. Philos. Mag. 30, 293-305 (1974).

38. Kramer, E. J. Scaling laws for flux pinning in hard superconductors. J. Appl. Phys. 44, 1360-1370 (1973).

\section{Acknowledgements}

We thank J. D. Thompson, Y. Bang, Z. Fisk, I. Vekhter, Y. Yang, J. H. Yun, and S. Oh for helpful discussions. This work was supported by a National Research Foundation (NRF) of Korea grant funded by the Korean Ministry of Science, ICT and Planning (No. 2012R1A3A2048816). S.-G.J. was supported by the Basic Science Research Program through the National Research Foundation of Korea (NRF) funded by the Ministry of Education (NRF-2015R1D1A1A01060382). Work at Los Alamos National Laboratory was performed under the auspices of the U.S. Department of Energy, Office of Basic Energy Sciences, Division of Materials Sciences and Engineering. S.S. acknowledges a Director's Postdoctoral Fellowship through the Los Alamos Laboratory Directed Research and Development program.

\section{Author contributions}

S.-G.J. conceived the work. S.-G.J., S.S., and S.L. performed the $I-V$ measurements at various pressures. E.D.B. and H.-O.L. synthesized the CeRhIn ${ }_{5}, \mathrm{Sn}_{\text {-doped }} \mathrm{CeRhIn}_{5}$, and $\mathrm{CeCoIn}_{5}$ single crystals. S.-G.J. analyzed the data and discussed the results with all authors. The manuscript was written by S.-G.J. and T.P. with inputs from all authors.

\section{Additional information}

Supplementary Information accompanies this paper at https://doi.org/10.1038/s41467 018-02899-5.

Competing interests: The authors declare no competing financial interests.

Reprints and permission information is available online at http://npg.nature.com/ reprintsandpermissions/

Publisher's note: Springer Nature remains neutral with regard to jurisdictional claims in published maps and institutional affiliations.

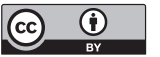

Open Access This article is licensed under a Creative Commons Attribution 4.0 International License, which permits use, sharing, adaptation, distribution and reproduction in any medium or format, as long as you give appropriate credit to the original author(s) and the source, provide a link to the Creative Commons license, and indicate if changes were made. The images or other third party material in this article are included in the article's Creative Commons license, unless indicated otherwise in a credit line to the material. If material is not included in the article's Creative Commons license and your intended use is not permitted by statutory regulation or exceeds the permitted use, you will need to obtain permission directly from the copyright holder. To view a copy of this license, visit http://creativecommons.org/ licenses/by/4.0/.

(C) The Author(s) 2018 